\title{
Intratumoral distribution of EGFR mutations and copy number in metastatic lung cancer, what impact on the initial molecular diagnosis?
}

\author{
Audrey Mansuet-Lupo 1,2,3,4, Fouzia Zouiti ${ }^{5}$, Marco Alifano ${ }^{6}$, Anne Tallet ${ }^{5}$, Marie-Christine Charpentier ${ }^{4}$, \\ Véronique Ducruit ${ }^{4}$, Fabrice Devez ${ }^{4}$, Fanny Lemaitre ${ }^{5}$, Pierre Laurent-Puig ${ }^{2,5,7}$, Diane Damotte ${ }^{1,2,3,4}$ \\ and Hélène Blons $2,5,7,8^{*}$
}

\begin{abstract}
Background: Activating epidermal growth factor receptor (EGFR) mutations characterize a subgroup of non-small-cell lung cancer that benefit from first line EGFR tyrosine kinase inhibitors (EGFR-TKI). However, the existence of polyclonal cell populations may hinder personalized-medicine strategies as patients' screening often depends upon a single tumor-biopsy sample. The purpose of this study is to clarify and to validate in clinical testing conditions the accuracy of EGFR genotyping using different tumor sites and various types of samples (transthoracic, surgical or endoscopic biopsies and cytology specimens).

Methods: We conducted a retrospective review of 357 consecutive patients addressed for EGFR mutation screening in accordance with the directive of the European Medicines Agency (stage IV NSCLC). Fifty-seven samples were EGFR mutated and 40 had adequate tumor specimens for analysis on multiple spatially separated sites. Ten wild type samples were also analyzed. A total of 153 and 39 tumor fragments, from mutated and non-mutated cases respectively, were generated to analyze tumor heterogeneity or primary-metastatic discordances. After histological review of all fragments, EGFR genotyping was assessed using the routine diagnostic tools: fragment analysis for insertions and deletions and allele specific TaqMan probes for point mutations. EGFR copy number (CN) was evaluated by qPCR using TaqMan probes.

Results: The identification of EGFR mutations was independent of localization within primary tumor, of specimen type and consistent between primary and metastases. At the opposite, for half of the samples, tumor loci showed different EGFR copy number that may affect mutation detection cut-off.

Conclusions: This is the largest series reporting multiple EGFR testing in Caucasians. It validates the accuracy of EGFR mutation screening from single tumor-biopsy samples before first line EGFR-TKI. The unpredictable variability in EGFR CN and therefore in EGFR wild type/mutant allelic ratio justifies the implementation of sensitive methods to identify patients with EGFR mutated tumors.
\end{abstract}

Keywords: EGFR mutation, Genetic heterogeneity, EGFR amplification

\footnotetext{
* Correspondence: helene.blons@parisdescartes.fr

¿Université Paris Descartes, Sorbonne, Paris cité, France

${ }^{5}$ Assistance Publique Hôpitaux de Paris, Department of Biology, Hôpital Européen Georges Pompidou, Paris, France

Full list of author information is available at the end of the article
} 


\section{Introduction}

Lung carcinoma is the first cause of death by cancer in the world, mostly because patients have an advanced stage disease at diagnosis [1]. Adenocarcinoma (ADC), the most frequent histological type is morphologically and biologically heterogeneous. Different architectural patterns have been described and different molecular pathways are involved in the carcinogenesis process [2]. Attention has largely been focused on proliferation pathways with the identification of mutations in oncogenes such as KRAS, EGFR, ALK, HER2, PI3KCA and BRAF that are potential or validated drug targets [3]. The first identified target in NSCLC was the EGF receptor. In 2004, EGFR activating mutations were identified in lung ADC and rapidly associated with response to EGFR-TKI $[4,5]$. Clinico-pathological features that correlate with these mutations include east-Asian ethnicity, adenocarcinoma histology, female sex, and never smoking history. In lung cancer the prevalence of EGFR mutations varies from $10 \%$ in Caucasians to more than $40 \%$ in Asian populations [6]. They are mainly located in the tyrosine kinase domain and $90 \%$ consist of either small deletions in exon 19 (DEL19) or a missense mutation in codon 21 that changes the leucine 858 in an arginine (p.L858R). Concerning rare alterations, about 3\% of the mutations occur at codon 719, resulting in the substitution of glycine by a cysteine, alanine or serine (p.G719X) or at codon 861 (p.L861Q) [7,8]. In addition, there are rare 1 to $2 \%$ in-frame insertion mutations in exon 20 [9]. The predictive value of frequent alterations (DEL19 and p. L858R) is more or less equivalent but some studies have reported a higher sensitivity and longer PFS for patients with DEL19 mutated NSCLC [10]. Concerning rare alterations, sensitivity to EFGR-TKI and PFS are globally lower [11]. In 2010, results from large phase III trial led to the restriction of EGFR tyrosine kinase inhibitors to EGFR mutated tumors in first line treatment [12]. EGFR mutational status has therefore became mandatory to determine which therapy will be the most appropriate to patients with stage IV diseases. In this context, genetic heterogeneity is an obstacle to correct determination of EGFR status on small biopsies specimens. Previous studies showed that the EGFR mutation status was discordant in different parts of the tumor or between primary or secondary metastatic sites [13-16]. At the opposite, Yatabe et al showed in a series of Asiatic patients that discordant cases where extremely rare [17] and it was suggested that discrepancies regarding EGFR mutations distribution could be due to methodological procedures [16-18]. If EGFR genotyping results depend on sample types it will negatively impact treatment decisions. In Caucasians, EGFR molecular status at various tumor sites remains to be examined in standard testing conditions to validate $E G F R$ molecular testing as a diagnostic tool. Finally, EGFR mutational heterogeneity could also explain the occurrence of secondary EGFR-TKI resistances. Patients undergoing EFGR-TKI treatments will ultimately relapse. Recurrences are related to various mechanisms among which the emergence of EGFR p. T790M clones seems to be the most frequent. This alteration, present as a minor sub-clone before treatment seems selected by EGFR-TKI treatments [19]. Most methods used for molecular diagnostic are not sensitive enough to detect minor p.T790M subclones $(<1 \%)$ and this alteration is rarely identified in untreated patients. Indeed, the reported frequency of baseline EGFR p.T790M mutations varies widely in the literature, ranging from $1 \%$ of all EGFR-mutant lung cancers [11] to $35 \%$ of all EGFRmutant lung cancers [20] and depends of the sensitivity of the assays used and their ability to identify minor clones within a tumor. The prognostic significance of baseline EGFR p.T790M has not been reported. In the acquired resistance setting, it has been demonstrated that the presence of p.T790M predicts a favorable prognosis and indolent progression, compared to the absence of p.T790M after TKI failure [19].

Because no large Caucasian series was tested for EGFR genetic heterogeneity, we addressed this question in clinical testing conditions thanks to a French nationwide EGFR mutation characterization program in advanced lung cancer (National Cancer Institute, INCa).

The aim of this study was to answer several questions of clinical relevance: -i- is EGFR mutation distribution, including p.T790M, heterogeneous within the primary tumor?; -ii- is EGFR mutation distribution heterogeneous between the primary tumor and thoracic metastases ?; -iii- Are microbiopsy or cytology samples suitable for EGFR screening? and iiii- is EGFR copy number heterogeneous within the tumor?

\section{Patients and methods \\ Patients}

We studied 357 consecutive patients with adenocarcinoma that had EGFR testing for clinical purpose at the Hôtel Dieu Hospital from January 2010 to June 2011 in accordance with the directive of the European Medicines Agency (stage IV NSCLC tested before EGFR-TKI treatment). We found that 57 patients out of 357 had EGFR mutated tumors (15.9\% of the entire series). For 40 patients we had enough tissues available for a multi-localization screening. No concomitant KRAS or HER2 alteration was identified on those samples. To rule out possible genotyping errors at time of diagnosis, we also tested 10 EGFR, KRAS and HER2 wild type (WT) adenocarcinomas. This study was reviewed and approved by the local ethic committee (Comité de Protection des Personnes/CPP 2012 0612). Patients' characteristics are shown in Table 1 and are in 


\begin{tabular}{ll}
$\begin{array}{l}\text { Table } \mathbf{1} \text { Clinical characteristics of patients with EGFR } \\
\text { mutated lung adenocarcinoma }\end{array}$ \\
\hline Gender & 13 \\
\hline Male & 27 \\
Female & Mean 64 [42 - 81] \\
Age (years) & \\
Tobacco status & 15 \\
S10 Pack per year & 14 \\
> 10 Pack per year & 11 \\
unknown & \\
TNM stage at initial diagnosis* & \\
I & 4 \\
II & 4 \\
III & 9 \\
IV & 20 \\
Unknown & 3 \\
\hline
\end{tabular}

Shows the clinical characteristics of patients with an EGFR mutated adenocarcinoma. Patients mentioned with stage 1 or 2 tumors have relapsed. EGFR testing was done using the available material (diagnostic samples) at time of relapse.

accordance with previous reports on EGFR mutated Caucasian series $[21,22]$.

\section{Samples}

All EGFR mutated adenocarcinomas had thyroid transcriptional factor (TTF1) nuclear expression (Novocastra, clone SPT24). The 22 surgical resections were classified according to the IASLC/ATS/ERS recommendation [23].

A total of 153 tumor fragments from 40 patients were selected by two pathologists (MLA and DD): 5 bronchial biopsies, 2 bronchial aspirations, 5 tomography-guided needle lung biopsies, 8 pleural effusions, 33 metastatic pleural localizations, 83 pulmonary surgical samples (lobectomy and wedge resections) and 17 lymph-nodes specimens. For each patient 2 to 19 loci were generated consisting of different localizations (primary and metastatic sites) or subdivision of the tumor tissue into small parts to analyze EGFR mutation at different sites, with different tumor cell content and different architectural patterns (Table 2, Additional file 1: Table S1). We called "small specimens": cytology (pleural effusions or bronchial aspiration) or small biopsy (tomography-guided needle lung biopsies or bronchial biopsies). Concerning wild type samples 39 fragments from 10 patients were generated, including 3 adrenal glands, 6 lymph-nodes, 2 pleural metastases and 28 fragments from the primary tumors. All contained more than $50 \%$ tumor cells to rule out possible mutant allele dilution (Additional file 2: Table S2).

In order to fit to the usual clinical practice of our pathology department, EGFR mutations were analyzed in a representative tumor area, without microdissection procedure. All samples (153 from EGFR mutated tumors, 39 from wild type samples) were reviewed for tumor cell content at x100 magnification (HES staining) by pathologists (MLA and DD) for 26 out of the 153 samples had low tumor cell contents (Additional file 1: Table S1 and Additional file 2: Table S2).

\section{Molecular analysis}

Genomic DNA was extracted from $20 \mu \mathrm{m}$-thick formalinfixed paraffin-embedded blocks using illustra ${ }^{\mathrm{Tm}}$ DNA extraction kit BACC2 (GE Healthcare), according to the manufacturer's instructions. EGFR mutations were analyzed using locally validated tests [24]. In the diagnostic setting, our screening strategy is to test EGFR exon 19 deletions (DEL19), exon 20 insertions (INS20) and the p.L858R mutation along with KRAS and HER2 exon 20 insertions. Non-mutated samples are subsequently analyzed for EGFR codons 719, 861 and mutated samples for EGFR p.T790M. The 153 fragments generated from mutated samples were analyzed for the mutation found at initial diagnosis, the 39 fragments from non-mutated cases were tested for the entire EGFR mutation panel (DEL19, INS20, p.L858R, p.L861Q and p.G719X).

Deletions and insertions were detected using fragment analysis with a FAM-labeled primer, run on an ABI 3730 XL (Applied biosystems, Foster City, CA) and analyzed with Genemapper software (Applied biosystems). Fragment analysis has a detection cut-off of $5-10 \%$ mutated/ wild type allele ratio. Samples with an expected DEL19 that were found wild-type $(n=3)$ using fragment analysis were subsequently re-analyzed by TaqMan ${ }^{\odot}$ Mutation Detection assays based on Competitive Allele Specific TaqMan PCR technology (CAST). Point mutations: p.L861Q, p.G719A, p.G719C, p.G719S, p.L858R and p.T790M were analyzed using similar technology. CAST probes were not available to test INS20 mutations. Allele specific assays were run in a final volume of $5 \mu \mathrm{l}$ in 384 wells plate including $2.5 \mu \mathrm{l}$ of 2X TaqMan ${ }^{\bullet}$ genotyping master mix (Applied Biosystems), $0.5 \mu \mathrm{l}$ of 10X Assay Mix (Hs00000173_rf :EGFR_rf; Hs00000141_mu :EGFR_6213_mu; Hs00000104_mu: EGFR_ 6239_mu; Hs00000148_mu: EGFR_6253_mu; Hs00000146_ mu: EGFR_6252_mu; Hs00000106_mu: EGFR_6240_mu; Hs00000102_mu: EGFR_6224_mu; Hs00000228_mu: EGFR ex19dels_mu from Lifetechnologies) and $1 \mu \mathrm{l}$ DNA template $(\leq 20 \mathrm{ng} / \mu \mathrm{l})$. Runs were performed in duplicates on an $\mathrm{ABI}$ Prism 7900 HT sequence detection system (Applied Biosystems) using the following thermo cycling conditions: $95^{\circ} \mathrm{C}$ / $10 \mathrm{~m}\left(92^{\circ} \mathrm{C} / 15 \mathrm{~s}, 58^{\circ} \mathrm{C} / 1 \mathrm{~m}\right)$ for 5 cycles, then $\left(92^{\circ} \mathrm{C} / 15 \mathrm{~s}\right.$, $60^{\circ} \mathrm{C} / 60 \mathrm{~s}$ ) for 40 cycles and analyzed with the SDS 2.0 software program (Applied Biosystems). qPCR analyses have a detection cut-off of 1-2\% mutated/wild type allele ratio [24]. Direct sequencing was run on a subset of samples as previously described [25]. 


\begin{tabular}{|c|c|c|c|c|c|c|c|c|c|}
\hline \multirow[b]{2}{*}{ Patients } & \multicolumn{5}{|c|}{ Number and type of samples analyzed per patients } & \multicolumn{4}{|c|}{ EGFR genotyping results } \\
\hline & Mutation type & $\begin{array}{l}\text { Primary tumor (sugical sample, } \\
\text { lung biopsy*, bronchial biospy } £ \text { ) }\end{array}$ & $\begin{array}{l}\text { PLEURAL } \\
\text { BIOPSY }\end{array}$ & $\begin{array}{l}\text { LYMPH } \\
\text { NODE }\end{array}$ & $\begin{array}{l}\text { Cytology: Pleural } \\
\text { Effusion, Bronchial } \\
\text { aspiration* }\end{array}$ & $\begin{array}{l}\text { Contributive } \\
\text { samples }\end{array}$ & $\begin{array}{l}\text { Concordant } \\
\text { EGFR status }\end{array}$ & $\begin{array}{l}\text { Non contributive } \\
\text { samples }\end{array}$ & Discordant EGFR status \\
\hline P1 & del19 & 7 & 0 & 0 & 0 & 7 & 7 & 0 & \\
\hline P2 & del19 & 2 & 0 & 0 & 0 & 2 & 2 & 0 & \\
\hline P3 & del19 & 0 & 3 & 0 & 1 & 4 & 3 & 0 & 1 (rescued by CAST PCR) \\
\hline P4 & del19 & 0 & 4 & 0 & 1 & 5 & 5 & 0 & \\
\hline P5 & del19 & 0 & 3 & 0 & 1 & 4 & 4 & 0 & \\
\hline P6 & del19 & $1^{*}+1^{*}$ & 0 & 0 & 0 & 2 & 2 & 0 & \\
\hline P7 & del19 & 0 & 0 & 0 & 2 & 2 & 2 & 0 & \\
\hline P8 & del19 & $1^{*}+1^{*}$ & 0 & 0 & 0 & 2 & 2 & 0 & \\
\hline P9 & del19 & 3 & 1 & 0 & 0 & 4 & 4 & 0 & \\
\hline P10 & del19 & 0 & 3 & 0 & 0 & 3 & 3 & 0 & \\
\hline P11 & del19 & 0 & 3 & 0 & 0 & 3 & 3 & 0 & \\
\hline P12 & del19 & 0 & 3 & 0 & 0 & 2 & 2 & 1 & \\
\hline P13 & del19 & 4 & 0 & 0 & 0 & 4 & 4 & 0 & \\
\hline P14 & del19 & 2 & 0 & 0 & 0 & 2 & 2 & 0 & \\
\hline P15 & del19 & 0 & 2 & 0 & 1 & 3 & 3 & 0 & \\
\hline P16 & del19 & $1 £+1 £$ & 0 & 0 & $1^{*}$ & 3 & 1 & 0 & 2 (remained WT by CAST PCR) \\
\hline P17 & del19 & 3 & 0 & 0 & 0 & 3 & 3 & 0 & \\
\hline P18 & del19 & 0 & 0 & 1 & $1^{*}$ & 1 & 1 & 1 & \\
\hline P19 & del19 & $1+1^{*}$ & 0 & 0 & 0 & 2 & 2 & 0 & \\
\hline P20 & p.G719A & 1 & 0 & 4 & 0 & 5 & 5 & 0 & \\
\hline P21 & p.G719A & 5 & 0 & 2 & 0 & 7 & 7 & 0 & \\
\hline P22 & ins20 & 3 & 0 & 1 & 0 & 4 & 4 & 0 & \\
\hline P23 & ins20 & 0 & 2 & 0 & 0 & 2 & 2 & 0 & \\
\hline P24 & ins20 & 3 & 0 & 2 & 0 & 4 & 4 & 1 & \\
\hline P25 & ins 20 & 0 & 2 & 0 & 0 & 2 & 2 & 0 & \\
\hline P26 & ins20 & 3 & 0 & 0 & 0 & 3 & 3 & 0 & \\
\hline P27 & ins20 & $2+1$ & 0 & $1+1$ & 0 & 4 & 2 & 1 & 2 \\
\hline P28 & p.L858R & 4 & 0 & 2 & 0 & 6 & 6 & 0 & \\
\hline P29 & p.L858R & $1 £+1 £$ & 0 & 0 & 0 & 2 & 2 & 0 & \\
\hline
\end{tabular}


Table 2 Multi-localization EGFR genotyping screening (Continued)

\begin{tabular}{|c|c|c|c|c|c|c|c|c|}
\hline P30 & p.L858R & 2 & 0 & 0 & 0 & 2 & 2 & 0 \\
\hline P31 & p.L858R & 3 & 0 & 0 & 0 & 3 & 3 & 0 \\
\hline P32 & p.L858R & 0 & 3 & 0 & 1 & 4 & 4 & 0 \\
\hline P33 & p.L858R & 0 & 3 & 0 & 0 & 3 & 3 & 0 \\
\hline P34 & p.L858R & $1 £$ & 1 & 0 & 1 & 3 & 3 & 0 \\
\hline P35 & p.L858R & 4 & 0 & 2 & 0 & 6 & 6 & 0 \\
\hline P36 & p.L858R & 2 & 0 & 1 & 0 & 3 & 3 & 0 \\
\hline P37 & p.L858R & 3 & 0 & 0 & 0 & 3 & 3 & 0 \\
\hline P38 & p.L858R & 3 & 0 & 0 & 0 & 3 & 3 & 0 \\
\hline P39 & p.L858R & 3 & 0 & 0 & 0 & 3 & 3 & 0 \\
\hline P40 & p.L861Q/p.T790M & 19 & 0 & 0 & 0 & 19 & 19 & 0 \\
\hline
\end{tabular}

Shows the type and number of samples tested for EGFR mutation in 40 patients. Non-contributive samples are non-amplified specimens. Discordant samples are DNAs that have been found wild type when a mutation was expected. The discordant samples are written in italic. Lung biopsy: *and bronchial biopsy: $£$. 


\section{EGFR copy number}

EGFR copy number was assessed by real time quantitative PCR using TaqMan ${ }^{\circ}$ copy number assays. Three probes were selected, in intron1, on intron7-exon8 and exon29-intron29 boundaries (Hs04960197_cn; Hs016463 07_cn; Hs00458616_cn). TaqMan ${ }^{\circ}$ Copy number reference assay RNase $\mathrm{P}$ was used as internal control and calibration was done using non-tumor formalin fixed paraffin embedded (FFPE) tissues. Samples were run in triplicates, the maximum difference tolerated between triplicates to calculate the average cycle threshold $(\mathrm{Ct})$ was 0.3 . EGFR copy number was given by the formula $2^{\text {-ddct }}$ as described previously [26]. Increased copy number was defined by a CNV $>2.5$. Cell lines with known copy number variations $(\mathrm{CNV})$ were used as controls. FFPE samples with RNase P Ct over 30 were not used for copy number quantification and qualified as non-contributive samples.

\section{Results}

1- EGFR mutation status on multiple spatially separated samples:

From January 2010 to March 2011, 357 patients managed at the Hotel-Dieu hospital for lung cancer had $E G F R$ testing for clinical purpose. Among the 57 EGFR mutated tumors, 40 patients had enough available tissue for multiple EGFR testing. Nineteen patients had an exon 19 deletion (DEL19), 12 a p.L858R point mutation, 6 an exon 20 insertion (INS20), 2 a p.G719A mutation and $1 \mathrm{a}$ p.L861Q/p.T790M double alteration. For those 40 patients, 153 DNA samples were extracted from various tumor localizations and reanalyzed for the initial alteration. Four samples could not be amplified (2 DEL19 and 2 INS20 tumors). The initial EGFR mutation was identified in 144 of the 149 informative samples. Five samples with an expected DEL19 $(\mathrm{n}=3)$ or INS20 $(\mathrm{n}=2)$ were found wild type. For those 5 cases, 2 cytologic specimens (pleural effusion and bronchial aspiration), 1 small endobronchial biopsy and 2 surgical specimens, the proportion of tumor cells was low, ranging from $2 \%$ to less than $10 \%$. One specimen (P3) was rescued by DEL19-CAST probes while both samples from P16 remained wild type (Table 2). CAST probes were not available to test INS20. There was no discordance for samples with expected $\mathrm{p}$. L858R, p.L861Q/p.T790M or p.G719A mutations (Table 2 and Additional file 1: Table S1).

All specimens were tested for the p.T790M mutation. This alteration was identified in one tumor (P40). Patient was naïve of EGFR-TKI, the tumor was p.L861Q/ p.T790M mutated, was divided into 19 parts and both mutations were homogeneously distributed with similar allele intensity (Additional file 1: Table S1). Samples that were initially diagnosed EGFR wild-type, were found wild-type on all sub-specimens (Additional file 2: Table S2).

2- Concordant EGFR mutation status was found within the tumor, and between primary and thoracic metastasis.

In our series, 22/40 patients had surgical resection for adenocarcinoma allowing classification according the IASLC/ATS/ERS recommendations [2]. All architectural patterns were represented except the mucinous pattern. Most tumors had a lepidic counterpart (9/22) but it was not necessary the predominant pattern (Additional file 1: Table S1). For these 22 adenocarcinomas, we analyzed different tumor area, displaying similar or different architectural patterns and no EGFR heterogeneity was seen within these specimens.

For 10 patients we analyzed both primary tumor and lymph node metastases $(n=8)$ or pleural metastases $(\mathrm{n}=2)$. A single discordant result was found between the primary tumor (INS20 mutation) and one metastatic lymph node containing $15 \%$ of tumor cells. For this patient, the mutation was present in the other lymph node metastasis (P27, Table 2, Additional file 1: Table S1).

Two patients showed a pre-invasive lesion (atypical adenomatous hyperplasia and in situ adenocarcinoma) located at distance of the invasive adenocarcinoma. These preinvasion lesions had the same EGFR mutation as the invasive counterpart (Additional file 1: Table S1).

3- Microbiopsy and cytology samples allow EGFR mutation analysis.

Twenty non-surgical specimens (8 pleural effusions, 2 bronchial aspirations, 5 tomography-guided needle lung biopsies, 5 bronchial biopsies) were analyzed including 8 with less than $15 \%$ tumor cells. Nineteen samples were contributive, 16 were EGFR mutated and 3 were wild type: 1 pleural effusion, 1 bronchial aspirate and 1 bronchial biopsy with 5, 5 and $10 \%$ tumor cell content respectively (Table 2 ).

4- Determination of EGFR copy number.

EGFR copy number (CN) was available in 132/153 samples. In this series, 26/132 samples had an EGFR $\mathrm{CN}>2.5$ and 2 an EGFR $\mathrm{CN}>5$. Half of the patients $(18 / 40)$ had at least one sample showing an increased EGFR CN but only one patient had homogeneous copy number increase on all fragment analyzed $(\mathrm{CN}>4$ on 3 pleural biopsy fragments, P11) (Table 3).

Concerning specimens with very low tumor cell content $(\leq 10 \%, n=25), 5$ were found wild type and 2 were non informative, none of these 7 specimens had an increased EGFR CN. At the opposite, among the mutated specimens with low tumor cell content $4 / 18$ had a $\mathrm{CN} \geq 2.5$ suggesting that $E G F R \mathrm{CN}$ impacts on mutation detection for low tumor cell content specimens.

We next examined whether increased EGFR CN was associated with specific morphologic features. In our 


\begin{tabular}{|c|c|c|c|c|c|c|c|c|}
\hline \multirow[b]{2}{*}{ Patients } & \multicolumn{5}{|c|}{$\begin{array}{l}\text { Number and type of samples } \\
\text { analyzed per patients }\end{array}$} & \multicolumn{3}{|c|}{ EGFR CNV results } \\
\hline & Mutation type & $\begin{array}{l}\text { Primary tumor (sugical sample, } \\
\text { lung biopsy*, bronchial biospy }\end{array}$ & $\begin{array}{l}\text { Pleural } \\
\text { biopsy }\end{array}$ & $\begin{array}{l}\text { Lymph } \\
\text { node }\end{array}$ & $\begin{array}{l}\text { Cytology: Pleural effusion, } \\
\text { Bronchial Aspiration* }\end{array}$ & $\begin{array}{l}\text { EGFR CNV } \\
\geq 2.5\end{array}$ & $\begin{array}{l}\text { EGFR CNV } \\
<2.5\end{array}$ & $\begin{array}{l}\text { Non contributive } \\
\text { samples }\end{array}$ \\
\hline P1 & del19 & 7 & 0 & 0 & 0 & 5 & 2 & \\
\hline P2 & del19 & 2 & 0 & 0 & 0 & & 2 & \\
\hline P3 & del19 & 0 & 3 & 0 & 1 & & 3 & 1 \\
\hline P4 & del19 & 0 & 4 & 0 & 1 & 1 & 3 & 1 \\
\hline P5 & del19 & 0 & 3 & 0 & 1 & 1 & 3 & \\
\hline P6 & del19 & $1^{*}+1^{*}$ & 0 & 0 & 0 & 1 & 1 & \\
\hline P7 & del19 & 0 & 0 & 0 & 2 & 1 & 1 & \\
\hline P8 & del19 & $1^{*}+1^{*}$ & 0 & 0 & 0 & 1 & 1 & \\
\hline P9 & del19 & 3 & 1 & 0 & 0 & 1 & 3 & \\
\hline P10 & del19 & 0 & 3 & 0 & 0 & & 1 & 2 \\
\hline P11 & del19 & 0 & 3 & 0 & 0 & 3 & & \\
\hline P12 & del19 & 0 & 3 & 0 & 0 & 1 & 2 & \\
\hline P13 & del19 & 4 & 0 & 0 & 0 & & 4 & \\
\hline P14 & del19 & 2 & 0 & 0 & 0 & & 2 & \\
\hline P15 & del19 & 0 & 2 & 0 & 1 & & 3 & \\
\hline P16 & del19 & $1 £+1 £$ & 0 & 0 & $1^{*}$ & & 1 & 2 \\
\hline P17 & del19 & 3 & 0 & 0 & 0 & 1 & 1 & 1 \\
\hline P18 & del19 & 0 & 0 & 1 & $1^{*}$ & & 1 & 1 \\
\hline P19 & del19 & $1+1^{*}$ & 0 & 0 & 0 & & 1 & 1 \\
\hline P20 & p.G719A & 1 & 0 & 4 & 0 & 1 & 2 & 2 \\
\hline P21 & p.G719A & 5 & 0 & 2 & 0 & & 6 & 1 \\
\hline P22 & ins20 & 3 & 0 & 1 & 0 & 2 & 2 & \\
\hline P23 & ins20 & 0 & 2 & 0 & 0 & & 2 & \\
\hline P24 & ins20 & 3 & 0 & 2 & 0 & & 4 & 1 \\
\hline P25 & ins20 & 0 & 2 & 0 & 0 & 1 & 1 & \\
\hline P26 & ins20 & 3 & 0 & 0 & 0 & & 3 & \\
\hline P27 & ins20 & $2+1$ & 0 & $1+1$ & 0 & & 4 & 1 \\
\hline P28 & p.L858R & 4 & 0 & 2 & 0 & & 6 & \\
\hline P29 & p.L858R & $1 £+1 £$ & 0 & 0 & 0 & 1 & 1 & \\
\hline P30 & p.L858R & 2 & 0 & 0 & 0 & & 2 & \\
\hline
\end{tabular}


Table 3 Multi-localization EGFR CNV screening (Continued)

\begin{tabular}{|c|c|c|c|c|c|c|c|c|}
\hline P31 & p.L858R & 3 & 0 & 0 & 0 & & 3 & \\
\hline P32 & p.L858R & 0 & 3 & 0 & 1 & 2 & 2 & \\
\hline P33 & p.L858R & 0 & 3 & 0 & 0 & 1 & 1 & 1 \\
\hline P34 & p.L858R & $1 £$ & 1 & 0 & 1 & 1 & & 2 \\
\hline P35 & p.L858R & 4 & 0 & 2 & 0 & & 6 & \\
\hline P36 & p.L858R & 2 & 0 & 1 & 0 & & 2 & 1 \\
\hline P37 & p.L858R & 3 & 0 & 0 & 0 & & 2 & 1 \\
\hline P38 & p.L858R & 3 & 0 & 0 & 0 & 1 & 2 & \\
\hline P39 & p.L858R & 3 & 0 & 0 & 0 & & 2 & 1 \\
\hline P40 & p.L861Q/p.T790M & 19 & 0 & 0 & 0 & & 18 & 1 \\
\hline
\end{tabular}

Shows the type and number of samples tested for EGFR copy number variation (CNV) in 40 patients. Non-contributive samples are non-amplified specimens or samples with Ct $>30$.

how 
experience, there was no clear association with highgrade lesions (solid or micropapillary predominant patterns) but in one patient, high EGFR CN was found in the solid pattern and not in the lepidic counterpart (Figure 1). At the opposite, 1 out of the EGFR mutated precursor lesions (in situ adenocarcinoma and atypical adenomatous hyperplasia), had an increased EGFR CN (CN: 3.4). No EGFR copy number increase was found in EGFR wild type tumor samples.

\section{Discussion}

Tyrosine kinase inhibitors (TKI) have changed advanced EGFR mutated lung carcinoma clinical practice with global improved short-term survival and fewer side effects [27]. In routine clinical practice EGFR mutation screening is mandatory to decide which first line treatment would be the most appropriate [28,29]. Heterogeneous repartition of EGFR mutations within tissue or between different metastatic sites is an obstacle to accurate molecular screening. It was reported in different studies and remains an important question for clinicians [13-15,30-32]. Table 4 summarizes previous works and compared samples and technologies. Main differences are, the inclusion of various cancer types including squamous-cell cancers that are not targets for EGFR-TKI treatments or pre-treated samples, different proportion of smokers, different types of metastatic site and finally different technologies and different panels of mutations tested. It seems that higher proportion of smokers, heterogeneous tumors specimens and the use of low sensitivity methods yielded to higher rates of inconsistencies in EGFR mutation results [33,34]. Moreover lower rates of concordance are also found for rare EGFR variants $[34,35]$. It seems therefore important to analyze in a series of patients prospectively tested for EGFR mutation in clinical settings, the impact of heterogeneity on diagnosis. Indeed, non-surgical specimens from either the primary or metastatic site often constitute the only tissue available for molecular diagnosis in patients eligible for TKI with advanced stage cancers $[13,30,32,36,37]$. Are these small specimens reliable for molecular testing? We addressed this question in Caucasian patients with EGFR mutated lung tumors. Multiple samples were obtained either from different localizations or different loci within the primary tumor. As expected in Caucasians, the frequency of EGFR mutated tumor was $15 \%$ [38]. The p.T790M resistance mutation was studied in all patients (153 specimens) that were

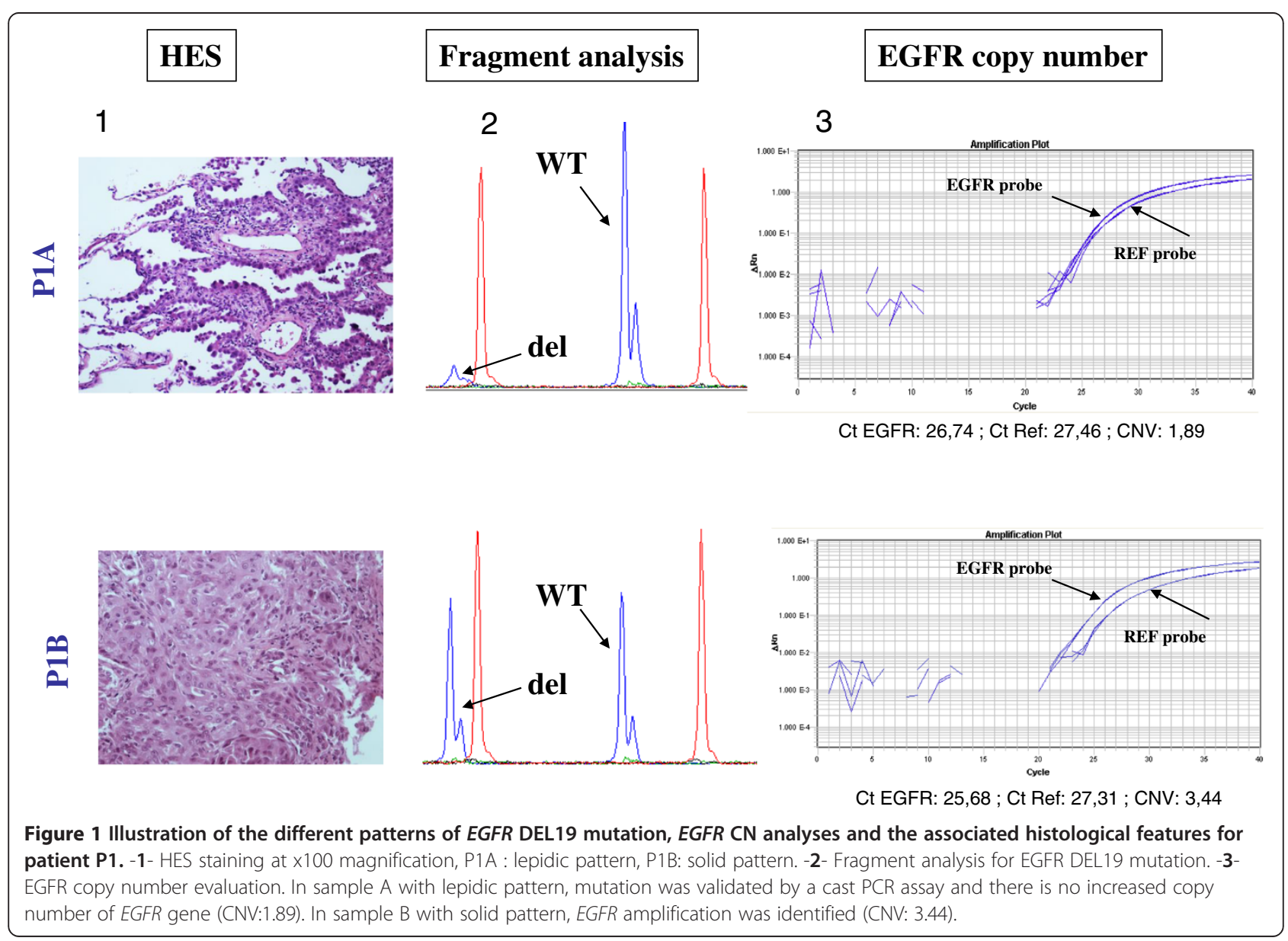


Table 4 Summary of previously published series

\begin{tabular}{|c|c|c|c|c|c|c|c|c|c|}
\hline Article & $\begin{array}{l}\text { Histological } \\
\text { subtype }\end{array}$ & No of samples & $\begin{array}{l}\text { Smoking } \\
\text { status }\end{array}$ & Concordance PT/M & Analysis within PT & $\begin{array}{l}\text { Concordance } \\
\text { within PT }\end{array}$ & Method & $\begin{array}{l}\text { Mutation } \\
\text { type }\end{array}$ & $\begin{array}{l}\text { Metastatic } \\
\text { sites }\end{array}$ \\
\hline \multirow{4}{*}{$\begin{array}{l}\text { Yatabe } \\
\text { et al. } 2011 \\
\text { [17] }\end{array}$} & \multirow[t]{4}{*}{$\mathrm{ADC}(77)$} & \multirow[t]{4}{*}{77} & & \multirow[t]{4}{*}{100} & & & $\mathrm{qPCR}$ & L858R & \multirow{4}{*}{$\begin{array}{l}\text { Lymph } \\
\text { nodes }\end{array}$} \\
\hline & & & & & & & \multirow[t]{3}{*}{ Fragment analysis } & DEL19 & \\
\hline & & & & & & & & INS20 & \\
\hline & & & & & & & & $719 X$ & \\
\hline \multirow[t]{3}{*}{$\begin{array}{l}\text { Sun et al. } \\
2011 \text { [37] }\end{array}$} & \multirow{3}{*}{$\begin{array}{l}\text { ADC (39), SCC } \\
\text { (31), ADSQ (6), } \\
\text { LCC (4) }\end{array}$} & \multirow[t]{3}{*}{80} & Ever (49) & & & & \multirow[t]{3}{*}{ Direct sequencing } & \multirow[t]{3}{*}{ all } & \multirow[t]{3}{*}{$\begin{array}{l}\text { Lymph } \\
\text { nodes }\end{array}$} \\
\hline & & & Never (31) & & & & & & \\
\hline & & & Global & $92,5 \%(74 / 80)$ & & & & & \\
\hline \multirow{4}{*}{$\begin{array}{l}\text { Wei et al. } \\
2014 \text { [41] }\end{array}$} & ADC (49) & \multirow[t]{4}{*}{50} & Ever (10) & $80 \%$ & & & \multirow{4}{*}{$\begin{array}{l}\text { qPCR (commercial } \\
\text { kit) }\end{array}$} & \multirow{4}{*}{$\begin{array}{l}45 \\
\text { hotspots }\end{array}$} & \multirow{4}{*}{$\begin{array}{l}\text { Lymph } \\
\text { nodes }\end{array}$} \\
\hline & $\operatorname{SCC}(1)$ & & & & & & & & \\
\hline & & & Never (40) & $97,5 \%$ & & & & & \\
\hline & & & Global & $93 \%(47 / 50)$ & & & & & \\
\hline \multirow{4}{*}{$\begin{array}{l}\text { Bai et al. } \\
2013 \text { [36] }\end{array}$} & ADC (63) & \multirow[t]{4}{*}{85 (45 EGFRmt 40 EGFRwt) } & & & 1431 foci & $87,1 \%$ & \multirow[t]{4}{*}{ ARMS } & \multirow{4}{*}{$\begin{array}{l}\text { DXS EGFR } \\
\text { mutation } \\
\text { Kit }\end{array}$} & \\
\hline & SCC (10) & & & & 1238 foci (foci : capture & 4 cases with $5 \%$ - & & & \\
\hline & ADSQ (5) & & & & $\begin{array}{l}\text { With laser } \\
\text { microdissection 0,1cm2) }\end{array}$ & $\begin{array}{l}8 \% \text { of foci showing } \\
\text { mutations }\end{array}$ & & & \\
\hline & Other (7) & & & & & & & & \\
\hline \multirow{5}{*}{$\begin{array}{l}\text { Chang et al. } \\
2011 \text { [42] }\end{array}$} & ADC (34) & \multirow[t]{5}{*}{56 (27 EGFRmt) } & Ever (29) & $62 \%$ & & & \multirow[t]{5}{*}{ Direct sequencing } & \multirow[t]{5}{*}{ all } & \multirow{5}{*}{$\begin{array}{l}\text { Lymph } \\
\text { nodes }\end{array}$} \\
\hline & SCC (17) & & & & & & & & \\
\hline & ADSQ (1) & & Never (23) & $70 \%$ & & & & & \\
\hline & \multirow[t]{2}{*}{ Other (1) } & & Unknown (4) & & & & & & \\
\hline & & & Global & $68 \%(38 / 56)$ & & & & & \\
\hline \multirow{4}{*}{$\begin{array}{l}\text { Schmid } \\
\text { et al. } 2009 \\
\text { [33] }\end{array}$} & \multirow[t]{4}{*}{ ADC (96) } & \multirow[t]{4}{*}{96 (7 EGFRmt) } & Ever (74) & & & & Direct sequencing & L858R (3) & Lymph \\
\hline & & & & & & & & DEL19 (3) & \\
\hline & & & Never (22) & & & & & INS20 (1) & \\
\hline & & & Global & $14 \%(1 / 7)$ & & & & & \\
\hline Gow et al & $\mathrm{ADC}(42)$ & 67 (35 EGFRmt) & Ever (26) & & & & Direct sequencing & all & Brain (25) \\
\hline 2009 [34] & SCC (21) & & & & & & discordant results & & Bone (20) \\
\hline & ADSQ (0) & (19 with adjuvant treatment & Never (41) & & & & & & Other (22) \\
\hline & Other (4) & $\begin{array}{l}\text { before molecular analysis on } \\
\text { metastatic site) }\end{array}$ & Global & $\begin{array}{l}26 \%(9 / 35) \text { seq and } 57 \% \\
(20 / 35) \text { ARMS }\end{array}$ & & & & & \\
\hline
\end{tabular}


Table 4 Summary of previously published series (Continued)

\begin{tabular}{|c|c|c|c|c|c|c|c|c|c|}
\hline $\begin{array}{l}\text { Mattsson } \\
\text { et al. } 2012 \\
{[18]}\end{array}$ & $\operatorname{ADC}(6)$ & 6 & & & $\begin{array}{l}3 \text { foci per tumor } \\
\text { (distinct } \\
\text { morphologies) }\end{array}$ & $100 \%$ & Direct sequencing & $\begin{array}{l}\text { L858R and } \\
\text { DEL19 }\end{array}$ & \\
\hline \multirow{7}{*}{$\begin{array}{l}\text { Kalikaki } \\
\text { et al. } 2008 \\
\text { [35] }\end{array}$} & ADC (20) & \multirow[t]{2}{*}{25 (7 EGFRmt) } & \multirow[t]{2}{*}{ Ever (22) } & & & & \multirow[t]{7}{*}{ Direct sequencing } & all & Brain (3) \\
\hline & $\operatorname{SCC}(2)$ & & & & & & & & Pleura (5) \\
\hline & ADSQ (0) & \multirow{5}{*}{$\begin{array}{l}\text { ( } 17 \text { with adjuvant treatment } \\
\text { before molecular analysis on } \\
\text { metastatic site) }\end{array}$} & \multicolumn{2}{|l|}{ Never (3) } & & & & & Lung (9) \\
\hline & \multirow[t]{4}{*}{ Other (3) } & & \multirow[t]{4}{*}{ Global } & $\begin{array}{l}14 \%(1 / 7) 5 \text { mutations are } \\
\text { rare alterations (codons } \\
692-847-746-857)\end{array}$ & & & & & $\begin{array}{l}\text { Adrenal } \\
\text { gland (3) }\end{array}$ \\
\hline & & & & & & & & & Bone (2) \\
\hline & & & & & & & & & Skin (1) \\
\hline & & & & & & & & & Liver (1) \\
\hline $\begin{array}{l}\text { Matsumoto } \\
\text { et al. } 2006 \\
\text { [43] }\end{array}$ & ADC (19) & 19 (12 EGFRmut) & & $100 \%$ & & & Direct sequencing & $\begin{array}{l}\text { L858R, } \\
\text { DEL19 }\end{array}$ & Brain (19) \\
\hline \multirow{3}{*}{$\begin{array}{l}\text { Yatabe } \\
\text { et al. } 2011 \\
{[17]}\end{array}$} & \multirow[t]{3}{*}{ ADC (50) } & \multirow[t]{3}{*}{50 EGFRmt } & & & \multirow[t]{2}{*}{3 foci per tumor (50) } & \multirow[t]{2}{*}{$100 \%$} & $q P C R$ & L858R & \\
\hline & & & & & & & Fragment analysis & DEL19 & \\
\hline & & & & & 100 foci per tumor (5) & $100 \%$ & & & \\
\hline
\end{tabular}

Schematic review of previously published series comparing primary tumor and metastasis or different loci within primary tumor. Tumor type, smoking status, detection methods, mutation tested and metastatic sites are given. PT : primary Tumor, M: metastasis, ADC: adenocarcinoma, SCC: squamous cell carcinoma, ADSQ : adenosquamous carcinoma, LCC: large cell carcinoma mt: mutated, wt : wild type, ARMS: amplification refractory mutation system. 
EGFR-TKI naïve. In untreated patient, the p.T790M mutation is usually described as a subclonal alteration that is difficult to identify even with highly sensitive methods [39]. Here the alteration was found once concomitantly to a p. L861Q mutation, both alleles were equally represented and the distribution was homogeneous suggesting that in this case, the p.T790M alteration is a driver mutation altogether with the p.L861Q. Routine EGFR testing gives a qualitative analysis of EGFR mutations, tumor is either mutated or non-mutated and the proportion of mutated allele is not taken into consideration. Using this definition, we found that 149/153 samples were contributive; among these 149 samples, only 5 showed a discordant genotype (WT/M). Discordant results were from low tumor cell content specimens expected to be either DEL19 or INS20. Fragment analysis, the method used to detect these alterations has a higher detection threshold as compared to allelic discrimination (10\% versus 1\%) [24], suggesting that these results might be false negative. Among the samples that could be tested using the DEL19 TaqMan ${ }^{\bullet}$ Mutation Detection assay one was found positive while both specimens from patient P16 remained negative. This patient had 2 primary cancers, concomitant adenocarcinoma and squamous cell cancer. Initial EGFR mutation was found on a bronchial biopsy sample showing an ADC, a second biopsy and the bronchial aspiration were found EGFR wild type even though high sensitivity CAST PCR was used. For this patient, the existence of 2 cancers might explain the presence of EGFR mutated and non-mutated samples. Indeed diagnosis on small specimens may be equivocal. To validate the accuracy of EGFR wild type status, 10 patients with a diagnosis of $E G F R$ wild type tumor were re-analyzed at different loci. No EGFR alteration was found (mutation or increased $\mathrm{CN}$ ). It suggests that negative results can be trusted as long as the method detection cut-off matches the tumor cell content. Although our work cannot rule out the existence of minor wild type subclones, we believe that any sample allows accurate EGFR mutations detection at initial diagnostic. This fits the results of Yatabe et al [17]. However, discrepancies may be increased by the use of low sensitivity methods such as sequencing. In this work, 38 samples were analyzed by direct sequencing. We found 23 concordances, 3 discordances and 12 informative sequences because of high background noise. We stopped the comparison as this method is not used in diagnosis in our lab and was shown to be inappropriate for biopsy or cytology FFPE samples. Indeed results depend on the estimation of the tumor cell content, on the method's detection cut-off and on EGFR copy number. What threshold of tumor nuclei should we use? It is assumed that more than $50 \%$ of tumor cells allows accurate molecular testing and that samples under 10\% may lead to false negative results [40]. Between 10\% and $50 \%$ the reliability depends on the laboratory experience. Interestingly, we had detectable EGFR mutations in $18 / 23$ contributive samples with $\leq 10 \%$ of tumor cells. It highlights the fact that if a positive result can be found in a sample with low tumor content, negative results have to be validated according to the specimen's tumor content. Pleural metastatic evolution is frequent in lung cancer and pleural effusion and/or tissue are convenient materials for molecular biology.

We secondly tested whether EGFR CN increase could explain why EGFR mutation assessment, remain possible for cases with very few tumor cells. We found that EGFR copy number was heterogeneous between different fragments from the same tumor. Thus, the allelic ratio of each specimen depends not only on the tumor cell content but also on the EGFR copy number and on the number of mutated allele in tumor cells. In clinical settings the proportion of EGFR mutated cells or the presence of an associated increased copy number, which is frequent in EGFR mutated tumors, is not taken into account. In samples with various tumor cell contents and various $E G F R$ gene copy number the quantification of the mutated allele/wild type allele ratio is difficult. It is clear to us that this ratio varies between tumor sites and that low tumor cell content specimens are rescued by the presence of a high mutant/wild type allele ratio. At the opposite, high tumor cell content specimens may show low mutant/wild type allele ratio estimated by the intensity of the mutant probe signal. This variability may explain discrepancies between series using different detection strategies or using microdissection of very few tumor cells as PCR amplifications in those conditions may lead to amplification errors [44]. However, it would be important to set up assays to analyze the allelic ratio's impact on response to treatment. One other question was the link between histology and EGFR mutation or $\mathrm{CN}$. In our experience, no difference in EGFR mutation status was found according to adenocarcinoma architectural patterns. This is in accordance with a report on a small series of samples $(\mathrm{n}=11)$ from a Swedish group [18]. As already reported EGFR copy number was variable within the same tumor and may vary according to the architectural pattern $[17,45]$. Here, for one tumor, EGFR increased copy number was restricted to the solid counterpart as compared to lepidic pattern. But this could not be generalized as other solid or micropapillary predominant pattern tumors had no EGFR $\mathrm{CN}$ increase and at the opposite a preinvasive lesion showed a high EGFR copy number. Finally, our results suggested that the role of EGFR amplification in cancer progression might not be directly linked to the histological tumor grade.

Patients undergoing EGFR TKI treatment will develop resistance after several months. Heterogeneity has been described as a phenomenon that could drive secondary resistance. Issues are either the development of a 
p.T790M or a wild type subclone $[15,30,39]$. In our series, there was no p.T790M heterogeneity. Considering that our detection method (CAST-PCR) can detect approximately 1 to $2 \%$ of mutated alleles in a background of wild type this might not have been sufficient for $\mathrm{p}$. T790M subclone detection [24]. It was also suggested that secondary resistances might be due to EGFR mutation loss. Our study was not designed to detect the presence of EGFR wild type subclones however, if this wild type tumor cell population exists, it is not an obstacle to initial EGFR molecular diagnosis defined by the qualitative presence of the EGFR mutation.

\section{Conclusions}

This retrospective series reports multiple EGFR testing in lung cancer in routine diagnostic conditions and validates that molecular testing from single tumor-biopsy sample before first line EGFR-TKI may be conducted on any specimens. This is an important result for clinical practice, it indicates that EGFR testing is relevant on biopsies, cytological samples, lymph nodes and metastatic sites using standardized and validated procedures with a defined detection cut-off. This existence of multiple primary tumors with possible distinct genotypes needs to be considered and the development of sensitive methods is recommended because the wild type/mutated allele ratio is unpredictable. Finally, the clinical impact of the associated EGFR copy number increase in a subset of samples remains to be evaluated.

\section{Additional files}

Additional file 1: Table S1. Detailed results showing multilocalisation screening for EGFR mutation and EGFR CNV.

Additional file 2: Table S2. Wild type sample results.

\section{Competing interest}

Following authors have declared that they do not have any conflict of interests: Mansuet-Lupo A, Zouiti F, Alifano M, Charpentier MC, Ducruit V, Devez F, Lemaitre F, Damotte D and Blons H Pierre Laurent-Puig declared being a consultant or advisory role for Amgen, Roche, Merck-Serono, Genomic Health, Astellas, Sanofi, Integragen and Raindance.

\begin{abstract}
Authors' contribution
AL carried out the molecular genetic studies, carried out tumor classification according to the IASLC/ATS/ERS recommendation, analyzed the data, and drafted the manuscript. FZ, FL carried out the molecular genetic studies, analyzed molecular data. MA recruited the patients, drafted the manuscript. CMC carried out tumor classification according to the IASLC/ATS/ERS recommendation. DV, DF carried out the pathology laboratory work. DM, PLP participated in the design of the study, performed analysis and helped to draft the manuscript. HB conceived of the study participated in its design and coordination and helped to draft the manuscript. All authors read and approved the final manuscript.
\end{abstract}

\section{Acknowledgements}

We thank The French National Cancer Institute (INCa) for its financial support (Plan cancer 2009-2013).

\section{Author details}

'UMRS 872, INSERM, Centre de Recherche des Cordeliers, Paris, France.

${ }^{2}$ Université Paris Descartes, Sorbonne, Paris cité, France. ${ }^{3}$ Université Pierre et Marie Curie, Paris, France. ${ }^{4}$ Assistance Publique Hôpitaux de Paris,

Department of Pathology, Hôpital Cochin-Hôtel Dieu, Paris, France.

${ }^{5}$ Assistance Publique Hôpitaux de Paris, Department of Biology, Hôpital Européen Georges Pompidou, Paris, France. ${ }^{6}$ Assistance Publique Hôpitaux de Paris, Department of Thoracic Surgery, Hôpital Cochin, Paris, France. ${ }^{7}$ UMR-S775, INSERM, Centre Universitaire des Saints Pères, Paris, France. ${ }^{8}$ INSERM, Université Paris Descartes, 45 Rue des Saints Pères, Paris 75006, France.

Received: 5 December 2013 Accepted: 7 April 2014

Published: 16 May 2014

\section{References}

1. Herbst RS, Heymach JV, Lippman SM: Lung cancer. N Engl J Med 2008 359:1367-1380

2. Yoshizawa A, Motoi N, Riely GJ, Sima CS, Gerald WL, Kris MG, Park BJ, Rusch W, Travis WD: Impact of proposed IASLC/ATS/ERS classification of lung adenocarcinoma: prognostic subgroups and implications for further revision of staging based on analysis of 514 stage I cases. Mod Pathol 2011, 24:653-664.

3. Coate LE, John T, Tsao MS, Shepherd FA: Molecular predictive and prognostic markers in non-small-cell lung cancer. Lancet Oncol 2009, 10:1001-1010.

4. Sordella R, Bell DW, Haber DA, Settleman J: Gefitinib-sensitizing EGFR mutations in lung cancer activate anti-apoptotic pathways. Science 2004, 305:1163-1167.

5. Lynch TJ, Bell DW, Sordella R, Gurubhagavatula S, Okimoto RA, Brannigan BW, Harris PL, Haserlat SM, Supko JG, Haluska FG, Louis DN, Christiani DC, Settleman J, Haber DA: Activating mutations in the epidermal growth factor receptor underlying responsiveness of non-small-cell lung cancer to gefitinib. N Engl J Med 2004, 350:2129-2139.

6. Subramanian J, Govindan R: Lung cancer in never smokers: a review. J Clin Oncol 2007, 25:561-570.

7. Mitsudomi T, Yatabe Y: Mutations of the epidermal growth factor receptor gene and related genes as determinants of epidermal growth factor receptor tyrosine kinase inhibitors sensitivity in lung cancer. Cancer Sci 2007, 98:1817-1824.

8. Yatabe $Y$, Mitsudomi T: Epidermal growth factor receptor mutations in lung cancers. Pathol Int 2007, 57:233-244

9. Arcila ME, Nafa K, Chaft JE, Rekhtman N, Lau C, Reva BA, Zakowski MF, Kris MG, Ladanyi M: EGFR exon 20 insertion mutations in lung adenocarcinomas: prevalence, molecular heterogeneity, and clinicopathologic characteristics. Mol Cancer Ther 2013, 12:220-229.

10. Jackman DM, Yeap BY, Sequist LV, Lindeman N, Holmes AJ, Joshi VA, Bell DW, Huberman MS, Halmos B, Rabin MS, Haber DA, Lynch TJ, Meyerson M, Johnson BE, Janne PA: Exon 19 deletion mutations of epidermal growth factor receptor are associated with prolonged survival in non-small cell lung cancer patients treated with gefitinib or erlotinib. Clin Cancer Res 2006, 12:3908-3914

11. Wu JY, Yu CJ, Chang YC, Yang CH, Shih JY, Yang PC: Effectiveness of tyrosine kinase inhibitors on "uncommon" epidermal growth factor receptor mutations of unknown clinical significance in non-small cell lung cancer. Clin Cancer Res 2011, 17:3812-3821.

12. Fukuoka $M, W u Y L$, Thongprasert $S$, Sunpaweravong $P$, Leong SS, Sriuranpong V, Chao TY, Nakagawa K, Chu DT, Saijo N, Duffield EL, Rukazenkov Y, Speake G, Jiang H, Armour AA, To KF, Yang JC, Mok TS: Biomarker analyses and final overall survival results from a phase III, randomized, open-label, first-line study of gefitinib versus carboplatin/ paclitaxel in clinically selected patients with advanced non-small-cell lung cancer in Asia (IPASS). J Clin Oncol 2011, 29:2866-2874.

13. Jiang SX, Yamashita K, Yamamoto M, Piao CJ, Umezawa A, Saegusa M, Yoshida T, Katagiri M, Masuda N, Hayakawa K, Okayasu I: EGFR genetic heterogeneity of nonsmall cell lung cancers contributing to acquired gefitinib resistance. Int J Cancer 2008, 123:2480-2486.

14. Sakurada A, Lara-Guerra H, Liu N, Shepherd FA, Tsao MS: Tissue heterogeneity of EGFR mutation in lung adenocarcinoma. J Thorac Oncol 2008, 3:527-529.

15. Taniguchi K, Okami J, Kodama K, Higashiyama M, Kato K: Intratumor heterogeneity of epidermal growth factor receptor mutations in lung cancer and its correlation to the response to gefitinib. Cancer Sci 2008, 99:929-935. 
16. Jakobsen JN, Sorensen JB: Intratumor heterogeneity and chemotherapy-induced changes in EGFR status in non-small cell lung cancer. Cancer Chemother Pharmacol 2012, 69:289-299.

17. Yatabe $Y$, Matsuo K, Mitsudomi T: Heterogeneous distribution of EGFR mutations is extremely rare in lung adenocarcinoma. J Clin Oncol 2011, 29:2972-2977.

18. Mattsson JS, Imgenberg-Kreuz J, Edlund K, Botling J, Micke P: Consistent mutation status within histologically heterogeneous lung cancer lesions. Histopathology 2011, 61:744-751.

19. Oxnard GR, Arcila ME, Sima CS, Riely GJ, Chmielecki J, Kris MG, Pao W Ladanyi M, Miller VA: Acquired resistance to EGFR tyrosine kinase inhibitors in EGFR-mutant lung cancer: distinct natural history of patients with tumors harboring the T790M mutation. Clin Cancer Res 2011, 17:1616-1622

20. Rosell R, Molina MA, Costa C, Simonetti S, Gimenez-Capitan A, Bertran-Alamillo J, Mayo C, Moran T, Mendez P, Cardenal F, Isla D, Provencio M, Cobo M, Insa A, Garcia-Campelo R, Reguart N, Majem M, Viteri S, Carcereny E, Porta R, Massuti B, Queralt C, De Aguirre I, Sanchez JM, Sanchez-Ronco M, Mate JL, Ariza A, Benlloch S, Sanchez JJ, Bivona TG, et al: Pretreatment EGFR T790M mutation and BRCA1 mRNA expression in erlotinib-treated advanced non-small-cell lung cancer patients with EGFR mutations. Clin Cancer Res 2011, 17:1160-1168.

21. Calvo $E$, Baselga J: Ethnic differences in response to epidermal growth factor receptor tyrosine kinase inhibitors. J Clin Oncol 2006, 24:2158-2163.

22. Pao W, Girard N: New driver mutations in non-small-cell lung cancer. Lancet Oncol 2011, 12:175-180.

23. Travis WD, Brambilla E, Noguchi M, Nicholson AG, Geisinger KR, Yatabe $Y$ Beer DG, Powell CA, Riely GJ, Van Schil PE, Garg K, Austin JH, Asamura H, Rusch WW, Hirsch FR, Scagliotti G, Mitsudomi T, Huber RM, Ishikawa Y, Jett J, Sanchez-Cespedes M, Sculier JP, Takahashi T, Tsuboi M, Vansteenkiste J, Wistuba I, Yang PC, Aberle D, Brambilla C, Flieder D, et al: International association for the study of lung cancer/american thoracic society/european respiratory society international multidisciplinary classification of lung adenocarcinoma. J Thorac Oncol 2011, 6:244-285

24. Didelot A, Le Corre D, Luscan A, Cazes A, Pallier K, Emile JF, Laurent-Puig P, Blons H: Competitive allele specific TaqMan PCR for KRAS, BRAF and EGFR mutation detection in clinical formalin fixed paraffin embedded samples. Exp Mol Pathol 2012, 92:275-280.

25. Pallier K, Houllier AM, Le Corre D, Cazes A, Laurent-Puig P, Blons H: No somatic genetic change in the paxillin gene in nonsmall-cell lung cancer. Mol Carcinog 2009, 48:581-585

26. Livak KJ, Schmittgen TD: Analysis of relative gene expression data using real-time quantitative PCR and the 2(-Delta Delta $C(T))$ Method. Method 2001, 25:402-408.

27. Sequist LV, Martins RG, Spigel D, Grunberg SM, Spira A, Janne PA, Joshi VA, McCollum D, Evans TL, Muzikansky A, Kuhlmann GL, Han M, Goldberg JS, Settleman J, lafrate AJ, Engelman JA, Haber DA, Johnson BE, Lynch TJ: First-line gefitinib in patients with advanced non-small-cell lung cancer harboring somatic EGFR mutations. J Clin Oncol 2008, 26:2442-2449.

28. Santarpia M, De Pas TM, Altavilla G, Spaggiari L, Rosell R: Moving towards molecular-guided treatments: erlotinib and clinical outcomes in non-small-cell lung cancer patients. Future Oncol 2013, 9:327-345.

29. Kobayashi K, Hagiwara K: Epidermal growth factor receptor (EGFR) mutation and personalized therapy in advanced nonsmall cell lung cancer (NSCLC). Target Oncol 2013, 8:27-33.

30. Chen ZY, Zhong WZ, Zhang XC, Su J, Yang XN, Chen ZH, Yang JJ, Zhou Q, Yan HH, An SJ, Chen HJ, Jiang BY, Mok TS, Wu YL: EGFR mutation heterogeneity and the mixed response to EGFR tyrosine kinase inhibitors of lung adenocarcinomas. Oncologist 2012, 17:978-985.

31. Honda Y, Takigawa N, Fushimi S, Ochi N, Kubo T, Ozaki S, Tanimoto M, Kiura $\mathrm{K}$ : Disappearance of an activated EGFR mutation after treatment with EGFR tyrosine kinase inhibitors. Lung Cancer 2012, 78:121-124.

32. Shimizu K, Yukawa T, Hirami Y, Okita R, Saisho S, Maeda A, Yasuda K, Nakata M: Heterogeneity of the EGFR mutation status between the primary tumor and metastatic lymph node and the sensitivity to EGFR tyrosine kinase inhibitor in non-small cell lung cancer. Target Oncol 2012, 8:237-242.

33. Schmid K, Oehl N, Wrba F, Pirker R, Pirker C, Filipits M: EGFR/KRAS/BRAF mutations in primary lung adenocarcinomas and corresponding locoregional lymph node metastases. Clin Cancer Res 2009, 15:4554-4560.

34. Gow CH, Chang YL, Hsu YC, Tsai MF, Wu CT, Yu CJ, Yang CH, Lee YC, Yang $P C$, Shih JY: Comparison of epidermal growth factor receptor mutations between primary and corresponding metastatic tumors in tyrosine kinase inhibitor-naive non-small-cell lung cancer. Ann Oncol 2009, 20:696-702

35. Kalikaki A, Koutsopoulos A, Trypaki M, Souglakos J, Stathopoulos E, Georgoulias V, Mavroudis D, Voutsina A: Comparison of EGFR and K-RAS gene status between primary tumours and corresponding metastases in NSCLC. Br J Cancer 2008, 99:923-929.

36. Bai H, Wang Z, Wang Y, Zhuo M, Zhou Q, Duan J, Yang L, Wu M, An T, Zhao J, Wang J: Detection and clinical significance of intratumoral EGFR mutational heterogeneity in Chinese patients with advanced non-small cell lung cancer. PLoS One 2013, 8:e54170.

37. Sun L, Zhang Q, Luan H, Zhan Z, Wang C, Sun B: Comparison of KRAS and EGFR gene status between primary non-small cell lung cancer and local lymph node metastases: implications for clinical practice. J Exp Clin Cancer Res 2011, 30:30

38. Goto K, Satouchi M, Ishii G, Nishio K, Hagiwara K, Mitsudomi T, Whiteley J, Donald E, McCormack R, Todo T: An evaluation study of EGFR mutation tests utilized for non-small-cell lung cancer in the diagnostic setting. Ann Oncol 2012, 23:2914-2919.

39. Ma C, Wei S, Song Y: T790M and acquired resistance of EGFR TKI: a literature review of clinical reports. J Thorac Dis 2011, 3:10-18.

40. Ellison G, Zhu G, Moulis A, Dearden S, Speake G, McCormack R: EGFR mutation testing in lung cancer: a review of available methods and their use for analysis of tumour tissue and cytology samples. J Clin Pathol 2012, 66:79-89.

41. Wei B, Yang K, Zhao J, Chang Y, Ma Z, Dong B, Guo Y, Ma J: Quantification of EGFR mutations in primary and metastatic tumors in non-small cell lung cancer. J Exp Clin Cancer Res 2014, 33:5.

42. Chang YL, Wu CT, Shih JY, Lee YC: Comparison of p53 and epiderma growth factor receptor gene status between primary tumors and lymph node metastases in non-small cell lung cancers. Ann Surg Oncol 2011, 18:543-50.

43. Matsumoto S, Takahashi K, Iwakawa R, Matsuno Y, Nakanishi Y, Kohno T, Shimizu E, Yokota J: Frequent EGFR mutations in brain metastases of lung adenocarcinoma. Int J Cancer 2006, 119:1491-4.

44. Akbari M, Hansen MD, Halgunset J, Skorpen F, Krokan HE: Low copy number DNA template can render polymerase chain reaction error prone in a sequence-dependent manner. J Mol Diagn 2005, 7:36-39.

45. Grob TJ, Hoenig T, Clauditz TS, Atanackovic D, Koenig AM, Vashist YK, Klose H, Simon R, Pantel K, Izbicki JR, Bokemeyer C, Sauter G, Wilczak W: Frequent intratumoral heterogeneity of EGFR gene copy gain in non-small cell lung cancer. Lung Cancer 2012, 79:221-227.

doi:10.1186/1479-5876-12-131

Cite this article as: Mansuet-Lupo et al:: Intratumoral distribution of EGFR mutations and copy number in metastatic lung cancer, what impact on the initial molecular diagnosis? Journal of Translational Medicine 2014 12:131.

\section{Submit your next manuscript to BioMed Central and take full advantage of:}

- Convenient online submission

- Thorough peer review

- No space constraints or color figure charges

- Immediate publication on acceptance

- Inclusion in PubMed, CAS, Scopus and Google Scholar

- Research which is freely available for redistribution 